\title{
DIE STANDÖRTLICHE VARIABILITÄT VON BÖDEN UND WASSERHAUSHALT IN HISTORISCHEN GÄRTEN UND KULTURLANDSCHAFTEN UND IHRE BEDEUTUNG FÜR DIE ANPASSUNG AN DEN KLIMAWANDEL
}

\begin{abstract}
Zur Struktur, Vielfalt und Entwicklung der Gehölzflora in historischen Gärten und Kulturlandschaften liegen in der Regel zahlreiche historische und aktuelle Dokumentationen vor. Im Gegensatz dazu fehlen entsprechende Informationen zur räumlichen und zeitlichen Variabilität von Böden und ihres Wasserhaushalts. Ein Vergleich der für den Schlosspark Branitz und das Dessau-Wörlitzer Gartenreich vorliegenden aktuellen Kartierungsergebnisse liefert hier wichtige Hinweise auf die große Heterogenität der Substrateigenschaften und die bodenhydrologischen Bedingungen. Hierbei zeigt sich, dass die aus natürlichen Prozessen der Landschafts- und Bodengenese hervorgegangenen Böden sowohl durch Vornutzung als auch durch gestalterische Eingriffe zum Teil stark überprägt sind. Für den Park Babelsberg fehlt eine solche hochaufgelöste Information. Diese Befunde verdeutlichen, dass eine gezielte und ressourceneffiziente Bewirtschaftung und Bestandsplanung nur möglich ist, wenn die Standortheterogenität und andere Aspekte räumlich hochaufgelöst erfasst und in einem weiteren Schritt mit modernen Methoden der Geodatenverarbeitung (GIS) abgebildet und analysiert werden. Mit Blick auf präventive Maßnahmen zur Klimaanpassung und die Abwehr von Schäden in akuten klimatischen Extremsituationen ist daher die flächenhafte Erfassung der bodenkundlichen und bodenhydrologischen Standortbedingungen für eine zukunftsorientierte gartenpflegerische Planung eine unabdingbare Grundvoraussetzung.
\end{abstract}

As a general rule numerous historical and recent documents are available on the structure, diversity and development of woody plant flora in historic gardens and cultural landscapes. In contrast, no such information is available on the spatial and temporal variability of soils and their soil water regime. A comparison of the current mapping results for the Schlosspark Branitz and the Dessau-Wörlitzer Gartenreich provides important information on the

Im Text werden Maskulinum und Femininum verwendet, wenn es um Personen geht. Gemeint sind grundsätzlich alle Menschen, gleich welcher Geschlechtsidentität sie sich zugehörig fühlen.

Ә OpenAccess. ๑ 2019 Bernd Uwe Schneider, Hans-Georg Frede, Knut Kaiser und Reinhard F. Hüttl, Berlin-Brandenburgische Akademie der Wissenschaften, publiziert von De Gruyter. (c) BY-NC-SA Dieses Werk ist lizenziert unter der Creative Commons AttributionNonCommercial-ShareAlike 4.0 Lizenz. https://doi.org/10.1515/9783110607482-016 
major heterogeneity of the substrate properties and the soil hydrology conditions. They reveal that the soils produced by natural processes of landscape and soil genesis are, in some cases, very much shaped by prior use on the one hand and design intervention on the other. In-depth information of this kind is not available for the Schlosspark Babelsberg. These results illustrate that targeted and resource-efficient management and vegetation planning is only possible if heterogeneity and other aspects are recorded in an in-depth manner and are depicted and analysed in a next step using modern geodata processing methods (GIS). With a view to preventive climate change adaptation measures and the warding off of damage in acute climate extreme situations, the comprehensive recording of pedological and hydrological site conditions is an essential precondition for future-oriented garden care planning.

\section{Klimatische Rahmenbedingungen im Untersuchungsraum}

Die historischen Gärten Großer Tiergarten Berlin, Babelsberg, Wörlitz und Branitz sind allesamt im Übergangsbereich vom ozeanisch zum kontinental geprägten Klima angesiedelt, der aufgrund geringer Höhendifferenzen nur wenig differenziert ist und zu den trockensten Regionen Deutschlands zählt. Die langjährigen mittleren Jahresniederschläge liegen bei 550 bis $600 \mathrm{~mm}$, wobei die Niederschläge in den Sommermonaten (Juni bis August) mit etwa $180 \mathrm{~mm}$ höher ausfallen als in den übrigen Jahreszeiten (Abb. 1). Der Deutsche Wetterdienst geht in seinen Klimaszenarien davon aus, dass die Jahresniederschläge für diese Region im Laufe des 21. Jahrhunderts zwar etwas zunehmen werden, das Mehr an Niederschlägen aber auf die Wintermonate konzentriert ist und dass die Niederschläge in den Sommermonaten eher abnehmen werden (https://www.dwd.de).

\section{Zur Historie der Bodenmelioration}

Die durch geringe Niederschläge vorgegebene ungünstige Wasserversorgung der vier historischen Gärten wird durch die jeweiligen physikalischen Bodeneigenschaften weiter verschlechtert, da die Gärten überwiegend auf sandigen Böden mit geringem Wasserspeichervermögen (synonym: nutzbare Feldkapazität) angelegt sind: Braunerden, Podsole und Pararendzinen aus Bauschutt (Großer Tiergarten), Pseudogley-Fahlerden und FahlerdePseudogleye aus Sand oder Lehmsand (Branitz), podsolige Braunerden und Podsol-Braunerden überwiegend aus Sand (Babelsberg) und Sande und Lehme über Sand (Wörlitz; vgl. Appel 2016).

Es gibt keinen Beleg dafür, dass diese ungünstigen hydrologischen Standortverhältnisse bei der Anlage der vier historischen Gärten als besonderes Problem angesehen wurden. Die Erfahrung zeigte vielmehr, dass leichte Böden für das Pflanzen und Gedeihen großer Bäume als vorteilhaft galten. Jäger (1865, 64 ff.) schreibt hierzu: »In anderen Fällen [gemeint ist das 


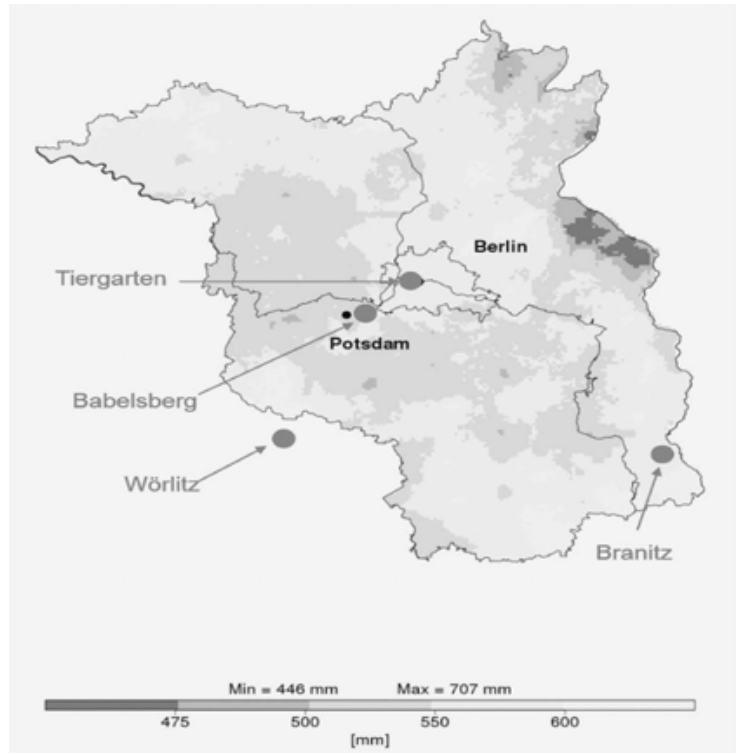

1 Langjährige Niederschläge in Brandenburg, Deutscher Klimaatlas 2018.

Pflanzen großer, einzeln stehender Bäume, Anm. d Verf.] sind die Kosten viel zu groß und die Erfolge nur auf Sandböden und anderem lockeren guten Boden günstig, während ich die Erfahrung machte, daß in schwerem Boden die meisten mit Sorgfalt behandelten Bäume nach 10 Jahren nur um weniges gewachsen waren, wovon nur Roßkastanien und nicht zu große Eichen eine günstige Ausnahme machten." Weiter heißt es bei Jäger (1865): »Die Anlagen des Fürsten Pückler in Muskau und Branitz, sowie einige in Potsdam und Umgebung von Lenné zeigen sehr günstige Erfolge. "Jäger beruft sich bei seinen Schilderungen auf eigene Erfahrungen, die eingeführten Verfahren von Meyer (1873) und weitere Autoren.

Um ein gutes Gedeihen von Pflanzungen auf leichten, sandigen Böden zu erreichen, wurden jedoch umfangreiche meliorative Maßnahmen ergriffen. Hierzu zählte zunächst das Rigolen (tiefgründiges Pflügen) der für die Pflanzungen vorgesehenen Flächen. Durch das Rigolen wurden »unregelmäßig geformte Flächen« (Jäger 1865) gelockert und homogenisiert und gegebenenfalls gutes Bodenmaterial aus dem Unterboden hochgepflügt. Auf das Rigolen wurde nur bei Einzelpflanzungen verzichtet. Hier wurden Pflanzgruben angelegt, die »im Durchmesser 2 bis $2 \frac{1}{2}$ Meter weiter und $1 / 2$ bis $3 / 4$ Meter tiefer sein [mussten], als der Wurzelballen des Baumes mißt [...] « (Meyer 1873, 230). Weiter heißt es dort: »Ferner muß die Pflanzgrube mit dem besseren Boden aus demselben, und ist dieser nicht außerordentlich fruchtbar, je nach Erfordernis mit Laub oder Mistbeeterde und mit fruchtbarem Lehm vermischt, so weit ausgefüllt werden, daß der Baum, wenn hineingestellt, mit den Wurzeln eher etwas über dem Rand der Grube hinaussteht, als nur um ein Weniges zu tief kommt." 
2 Rekonstruktion einer »Baummaschine» nach Hermann Fürst von Pückler-Muskau für den Transport und das Verpflanzen großer Bäume.

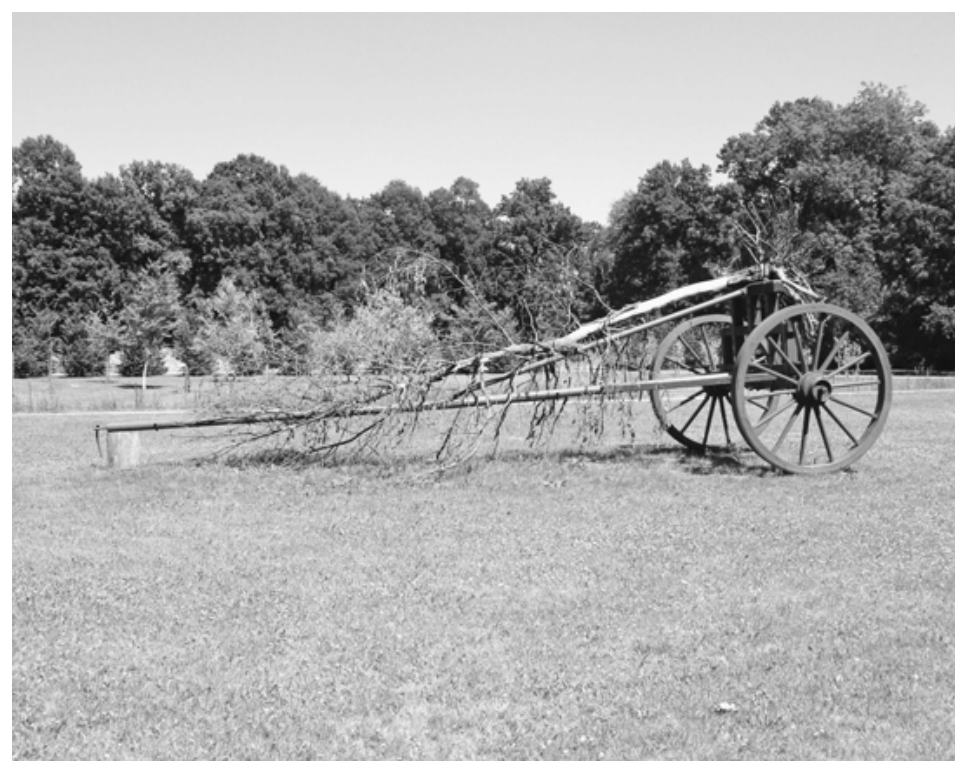

Die Pflanzgrube sollte schließlich eine Rampe aufweisen, um den Baum mit dem »Verpflanzwagen« (auch »Baummaschine« genannt), der speziell für die jeweilige Baumgröße dimensioniert war (Abb. 2), schonend in der Pflanzgrube platzieren zu können.

Nach dem Einsetzen des Baumes wurde Erde schonend »mit der Faust zwischen die Wurzeln und unter den Stamm « gestopft (Meyer 1873, 231), um die einzelnen Wurzeln in guten Kontakt mit dem Bodenmaterial zu bringen und damit eine ausreichende Wasserversorgung zu gewährleisten. Ein Einschlämmen erfolgte nach dem gezielten Einbringen des restlichen Bodenmaterials. Im Jahr der Anpflanzung sollten die Bäume mehrmals gewässert werden, wobei ein bis 8 Zoll $(\mathrm{ca} .20 \mathrm{~cm}$ ) hoher Bodenwall vom Durchmesser der Pflanzgrube das zugeführte Wasser auf den Wurzelbereich konzentrieren sollte (Meyer 1873, 231). Eine darüber hinausgehende langfristige Bewässerung war nicht vorgesehen. Stattdessen wurde großer Wert darauf gelegt, dass im Umkreis um den Baum eine Verdunstung von der Bodenoberfläche und damit eine Wasserkonkurrenz vermieden wurde:

"Eine gute Bedeckung des Bodens schützt vorm Austrocknen, und befördert so den Wuchs der Gehölze ungemein. Die beste Decke des Bodens ist das abgefallene Laub unter dem Schatten der Zweige. Dann folgt in Nützlichkeit eine Benarbung des Bodens durch niedrige, flachwurzelnde, genügsame Pflanzen. Sehr nachtheilig aber und den Boden erschöpfend wirken namentlich die tiefwurzenden, aber auch alle viel zehrende Unkräuter. Diese müssen überall vertilgt werden, wo sie sich nur blicken lassen [...].« (Fintelmann 1841, 202)

Die bei den Pflanzungen zu berücksichtigenden Maßnahmen wurden allgemein als außerordentlich bedeutsam für die Entwicklung der einzelnen Bäume und der Gesamtanlage 
angesehen. Meyer $(1873,228)$ fasst die Bedeutung dieser Tätigkeiten mit folgenden Worten zusammen:

»Deswegen ist es ein großer Irrthum, dafür zu halten, der Planausführer dürfe viele viel weniger künstlerische Begabung haben als der Planentwerfer: denn dieser giebt die allgemeinen Ideen her, jener aber soll sie richtig aufzufassen vermögen und fähig sein, sie weiter ins Einzelne zu entwickeln, was nur bei einem gewissen Grade von Kunstfertigkeit möglich ist.«

Zusammenfassend kann man sagen, dass sich eine ausreichende Wasserversorgung neuer Pflanzungen auf gezielte Maßnahmen vor und bei der Pflanzung konzentrierte (Rigolen, Bodenmaterial im Wurzelraum platzieren, Einschlämmen, Bewässern im Pflanzjahr, Verhinderung von Wasserkonkurrenz). Langfristige Bewässerung war dagegen von untergeordneter Bedeutung oder wurde in der Regel nicht in Erwägung gezogen.

Vor dem Hintergrund der jeweils durchgeführten Maßnahmen beim Pflanzen der Bäume können historische Gärten auch als Stätten zeitgenössischer kulturtechnischer Meliorationsmaßnahmen betrachtet werden. Es bleibt zu hinterfragen, ob die durchgeführten Maßnahmen unter den Bedingungen des sich abzeichnenden Klimawandels ausreichen werden, um die Pflanzungen zu schützen und weiterzuentwickeln.

$\mathrm{Zu}$ den verschiedenartigen Ausprägungen des Klimawandels gehören die Zunahme von Trockenperioden in der Wachstumszeit, extreme Sommertemperaturen, die Verschiebung der Hauptniederschlagszeit in die Wintermonate sowie sich häufende Starkregenereignisse und Stürme (Cubasch/Kadow 2011). Insbesondere ausgeprägte Trockenperioden in der Vegetationszeit, sommerliche Temperaturextreme, aber auch lange Stauwasserperioden sowie außergewöhnlich an- oder absteigendes Grundwasser sind geeignet, die Stabilität von Baumbeständen und Sträuchern und damit den denkmalpflegerischen Erhalt historischer Gärten nachhaltig zu beeinträchtigen. Für die Stabilität und Anpassungsfähigkeit der Gehölzflora an klimatische Extreme spielen - so wie in anderen Nutzungssystemen auch die Standorteigenschaften und hier vor allem die Böden eine zentrale Rolle. Von den Bodeneigenschaften hängt die Fähigkeit eines Standortes ab, Wasser und Nährstoffe zu speichern und für das pflanzliche Wachstum verfügbar zu machen. Diese Eigenschaften können je nach Standort erheblich variieren und beeinflussen so die Eignung eines Ortes für bestimmte Pflanzenarten und insbesondere die Vulnerabilität des Pflanzenbestandes gegenüber klimabedingten Stresssituationen. Zu den wichtigsten Einflussgrößen auf die Wasser- und Nährstoffverfügbarkeit eines Standorts zählen die Bodentextur (Korngrößenverteilung), der Mineralbestand, der Gehalt an stabiler organischer Bodensubstanz und die Mächtigkeit des durchwurzelbaren Bodens. Die Durchwurzelbarkeit ist oft durch anthropogene oder natürlich verursachte Verdichtung oder durch hoch anstehendes Grundwasser eingeschränkt. Eine Destabilisierung von Gehölzen kann also auch hydrologische Ursachen haben. Bei den hier behandelten Parkanlagen ist zu beachten, dass das Relief des Geländes durch intensive Aufschichtung von Böden, z.B. bei der Anlage von Hügeln, verändert wurde und so vergleichsweise junge, d.h. wenig entwickelte Böden (Lockersyroseme, Regsole etc.) entstanden sind, ohne die typischen morphologischen Merkmale und Eigenschaften natürlich entwickelter Böden. 
Die hier genannten Einflussgrößen vermitteln einen Eindruck von der Vielfalt relevanter Einflussparameter und den zwischen ihnen bestehenden Wechselwirkungen. Sie müssen bekannt sein, um die Klimaresilienz historischer Gärten und Kulturlandschaften einschätzen und daraus fundierte Handlungsoptionen für die Anpassung und den Schutz von Gehölzanlagen ableiten zu können. In der vorliegenden Arbeit soll daher für die im Rahmen der interdisziplinären Arbeitsgruppe »Historische Gärten im Klimawandel« ausgewählten Gärten in Babelsberg, Wörlitz und Branitz unter Nutzung von Kartierungsergebnissen eine vergleichende Bestandsaufnahme hinsichtlich der bodenkundlichen und bodenhydrologischen Standortbedingungen versucht werden. Der Vergleich soll Aufschluss geben, wie sich diese Standorte bodensystematisch und bodenhydrologisch unterscheiden und welche Hinweise für die Anfälligkeit gegenüber klimatischen Extremsituationen bzw. das Anpassungsvermögen der Gehölzflora an daraus abgeleitet werden können. Nachfolgend werden die einzelnen Standorte mit ihren jeweiligen Charakteristika vorgestellt.

\section{Das Dessau-Wörlitzer Gartenreich}

Für das Dessau-Wörlitzer Gartenreich wurden umfangreiche bodenkundliche und bodenhydrologische Untersuchungen mit dem Ziel durchführt, eine Gefährdungsanalyse für den Gehölzbestand dieser Kulturlandschaft vorzulegen (Kulturstiftung DessauWörlitz 2005). Eine Besonderheit dieses in und an der Elbeaue gelegenen Standorts ist das Mosaik aus teils grundwassernahen und somit hydromorph geprägten, teils grundwasserfernen Böden sowie die Exposition des Parks gegenüber periodischen Überschwemmungen durch die Elbe.

Die Mächtigkeiten der anhydromorphen Oberbodenhorizonte schwanken zwischen $>8 \mathrm{dm}$ und $<4 \mathrm{dm}$. In der Tabelle 1 sind die im Dessau-Wörlitzer Gartenreich vorherrschenden Bodensubtypen mit ihren Flächenanteilen (nur für Flächenanteile $\geq 5 \%$ ) aufgeführt.

Auf fast zwei Dritteln der kartierten Fläche konnten die Subtypen Gley-Vega und Vega-Gley ausgeschieden werden. Nennenswerte Flächenanteile entfallen ferner auf die Subtypen Gley-Regosol, Auengley und Gley-Kolluvisol. Damit sind über 80 \% der kartierten Fläche hydromorph, also von periodisch hoch anstehendem Grundwasser beeinflusst. In diesen Bodentypen dominieren die Substrattypen Lehm über Ton mit 27,9\%, Lehm über Sand mit 22,7\%, Lehmsand mit $19 \%$ sowie Lehmsand über Lehm mit 10\% des Flächenanteils. Aus diesen Befunden lässt sich ableiten, dass die Bodenentwicklung im Dessau-Wörlitzer Gartenreich von periodischen Überschwemmungen und damit einhergehenden - teilweise organischen - nährstoffreichen Sedimentablagerungen in Form von Auenlehmen, -schluffen und -tonen geprägt ist und dass die Mächtigkeit des anhydromorphen und damit durchwurzelbaren Bodens durch den Grundwasserstand erheblich beeinflusst wird. Einerseits gewährleisten hohe Grundwasserstände gerade in Trockenperioden die Wasserversorgung der Gehölzflora. Anderseits resultieren aus der intensiven Durchwurzelung der anhydromorphen Bodenhorizonte in aller Regel flach ausstreichende Wur- 


\begin{tabular}{l|l|l}
\hline Bodentyp & Fläche (ha) & Fläche (\%) \\
\hline Norm-Regosol $^{1}$ & 3,8 & 5,0 \\
\hline Gley-Regosol $^{2}$ & 10,1 & 13,3 \\
\hline Gley-Kolluvisol $^{3}$ & 4,9 & 6,5 \\
\hline Gley-Vega $^{4}$ & 21,4 & 28,1 \\
\hline Auengley & 8,7 & 11,4 \\
\hline Vega-Gley & 18,1 & 23,8 \\
\hline Andere/anthropogene Böden & 9,0 & 11,8 \\
\hline
\end{tabular}

Tabelle 1 Flächenanteile von Bodensubtypen im Dessau-Wörlitzer Gartenreich (Kulturstiftung DessauWörlitz 2005, 24). Die Bodenterminologie (siehe auch die Fußnoten) folgt der Bodenkundlichen Kartieranleitung/ KA5 (Ad-hoc-AG Boden 2005)

zelsysteme. Damit steigt die Anfälligkeit der Baumbestände für Windwürfe. Gleichzeitig kommt es durch die intensive Durchwurzelung des nicht von Grund- oder Stauwasser beeinflussten Oberbodens zu einem starken Nährstoffentzug in den entsprechenden Horizonten. Für diesen Standort sowie für viele andere Gärten und Kulturlandschaften gilt, dass der Nährstoffentzug im Rahmen der Parkpflege auf das Entfernen des Laubs im Herbst zurückzuführen ist. Hierfür sprechen auch die ausgewiesene geringe Kationenaustauschkapazität und der niedrige $\mathrm{pH}$-Wert der untersuchten Böden (Kulturstiftung DessauWörlitz 2005). An dieser Stelle kann nicht abschließend geklärt werden, welche Rolle der jahrzehntelange Eintrag basischer Stäube aus dem Betrieb nahegelegener Braunkohlekraftwerke für die Pflanzenernährung gespielt hat und wie sich der nunmehr ausbleibende Staubeintrag aktuell auf die Ernährung der Bestände auswirkt (Hüttl et al. 1995). Ein weiteres Risiko der hohen Wurzeldichte kann in ausgeprägten Trockenperioden zu Tage treten, wenn es zu einer raschen Grundwasserabsenkung kommen sollte. In diesen Phasen kommt es gerade wegen der hohen Wurzeldichte zu einer kurzfristigen Austrocknung des Oberbodens. In diesen Phasen können Bäume nicht unverzüglich mit verstärktem Wurzelwachstum reagieren oder wegen der in Gley-Böden oft vorherrschenden Verdichtung oder anoxischen Bedingungen nicht rechtzeitig in wasserführende Schichten vordringen. Die Folge ist eine potentiell unzureichende Wasserversorgung der Gehölze.

1 Regosol, von griechisch rhegos, Decke, bedecken; flachgründiger Boden im frühen Stadium der Bodenentwicklung mit zwei Horizonten (humoser Ober- und mineralischer Unterboden).

2 Gley, aus dem Westgermanischen: klaija, Lehm, Ton; grundwasserbeeinflusster Boden.

3 Kolluvisol, aus dem Lateinischen: das Zusammengeschwemmte; Böden aus durch Abschwemmung verlagertem, humosem Bodenmaterial (meist anthropogen verursacht).

4 Vega, aus dem Spanischen: fruchtbare Ebene; ein Bodentyp, der aus sedimentärem braunem Ausgangsmaterial im regelmäßig überfluteten Auenbereich von Flüssen entsteht. 


\section{Der Fürst-Pückler-Park Branitz}

Für diesen Park und weitere im Südosten Brandenburgs gelegene historische Gärten legten Rippl et al. (2013) ein detailreiches Kompendium mit Untersuchungsergebnissen zu bodenkundlichen, hydrologischen und die Pflanzenvitalität betreffenden Parametern vor. Anlass dieser Untersuchungen war die bergbauliche Planung zur möglichen Eröffnung des Tagebaus Cottbus-Süd, der nicht nur den Wasserhaushalt des Gartenareals betroffen, sondern auch Teile des Branitzer Parkgeländes in Anspruch genommen hätte. Wegen des zu erwartenden Eingriffs in das Grundwassersystem wurde eine Untersuchung initiiert, die die Grundwasserabsenkung und ihre möglichen Folgen abschätzen sollte. Die dabei auf Grundlage einer großmaßstäblichen Kartierung gewonnenen Ergebnisse zeigen, dass die Substrate durch historische und rezente Eingriffe anthropogen stark überprägt sind, wodurch die vorherrschenden Fein- und Mittelsande quartären (eiszeitlichen) Ursprungs oft mit bindigem Material durchmischt wurden oder aber Auffüllungen mit anderem Fremdmaterial erfolgten, die lokal den Grundwasserabstand erhöhten.

Die Ausgangssubstrate für die Bodenbildung im Branitzer Park sind holozäne (nacheiszeitliche) Bildungen und Grundmoränenablagerungen als Geschiebelehme und -mergel sowie Auelehme (50-70 \% Schluff und Ton, 20-40 \% Sande, 1-10 \% Kiese und Steine). Von Südwest nach Nordost durchzieht pleistozäner Beckenschluff den Park. Die Lehmschicht (40-60 cm) der Grundmoränenablagerungen ist häufig entkalkt, darunter ist kalkhaltiger Geschiebemergel nachgewiesen. Die Flusssande sind arm, weil sie keine Ton- und Schluffbeimengungen besitzen. Diesen Nachteil gleicht das durchgängig hochliegende Grundwasser aus.

Seltener sind Stagnogleye anzutreffen (Pücklerallee), die an stark von Stauwasser beeinflusste oberflächennahe Grundmoränen gebunden sind. Das kleinflächige Vorkommen der oberflächennahen Altmoräne der Saale-Eiszeit bedingt das höchste Nährstoff- und damit Ertragsniveau im Branitzer Park (Eichen der Pücklerallee und Schmiedewiese) im Vergleich zu den restlichen Arealen. Der Boden der Schmiedewiese hat einen doppelten Staukörper. Diese Besonderheit ist durch die Überströmung der Grundmoräne durch fließendes Wasser der Spree entstanden. In Stillwasserzeiten hat sich Auelehm über Geschiebelehm abgelagert.

Das Ertragsniveau der Bäume steigt vom Talbecken zum Moränensand über humose und bindige Gemische zu Geschiebelehmsand an (Rippl et al. 2013). Bei wasserbaulichen Arbeiten sind künstliche Hügelaufschüttungen aus Gewässeraushub entstanden. Sie bestehen aus grundwasserfreien Rohböden der örtlichen Substratmischung mit einem geringmächtigen Oberboden aus Laub- und Nadelstreu. Geländeunebenheiten wurden punktuell ausgeglichen.

Nach Rippl et al. (2013) gab es in der Branitzer Parkanlage ein besonderes Verfahren, bei dem ihr Gestalter Hermann Fürst von Pückler-Muskau Pflanzgruben mit Tierkadavern vorbereiten ließ, um die Bodenvitalität und -fruchtbarkeit zu steigern.

Stellenweise sind Erdmischungen mit wachstumsfördernden Eigenschaften vorhanden, z.B. östlich des Reitwegs, wo Rippl et al. (2013) bis in $80 \mathrm{~cm}$ Tiefe humose, kiesführende 


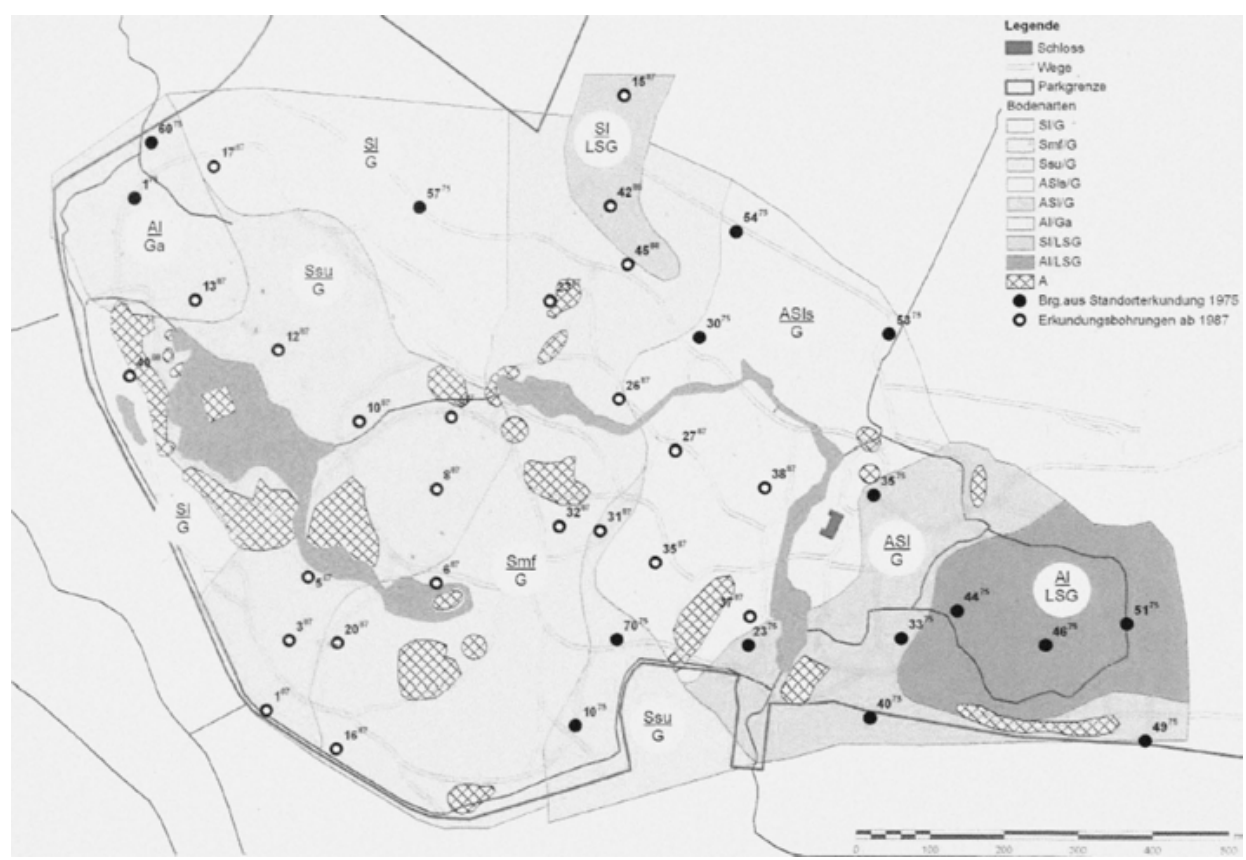

3 Substrattypen im Bereich von Park Branitz in Cottbus (Rippl et al. 2013, 178). Legendenschlüssel: $\mathrm{Sl}$ = Sande, schwach schluffig bis fein- und mittelkörnig; Smf = Sande, mittel- und feinkörnig; Ssu = Sande, stark lehmig und schluffig bis mittel- und feinkörnig; $\mathrm{Al}=$ Auelehm.

Grob- und Mittelsande mit kalkhaltigem Mergel nachwiesen. Diese Untersuchungen spiegeln sowohl die Eingriffe bei der Gestaltung des Parks als auch die vor seiner Gründung vorherrschende natürliche Bodengenese wider. Die Abbildung 3 gibt eine Übersicht über die im Park gefundenen Substrattypen (vgl. Appel 2016). Die Grundwasserstände liegen im Mittel bei $2 \mathrm{~m}$ Tiefe. Auf die für Gleye typischen morphologischen Merkmale trifft man daher erst in über $1 \mathrm{~m}$ Bodentiefe. Die wechselnden bindigen Beimengungen, die gut durchwurzelbaren Sande und das in dieser Tiefe anstehende Grundwasser haben sich unter den gegebenen klimatischen Bedingungen sehr förderlich auf das Baumwachstum ausgewirkt.

Diese Befunde verdeutlichen, dass an diesem Standort sowohl durch eine rasche Absenkung als auch durch den Anstieg des Grundwasserspiegels die Versorgung der Bäume mit Wasser beeinträchtigt werden kann. Aufgrund ihrer bodenkundlichen und hydrologischen Untersuchungen gelangen Rippl et al. (2013) zu dem Schluss, dass die Wasserverfügbarkeit wesentlich durch das regionale Niederschlagsgeschehen und durch Zuleitungen aus der Spree und aus Nebengerinnen bestimmt wird. Eine maßgebliche Beeinträchtigung des Bodenwasserhaushaltes durch den geplanten Tagebaubetrieb schlossen die Autoren seinerzeit aus. Die erwähnten Zuleitungen von Spreewasser zeigen aber auch, dass es in den vergangenen Jahrzehnten immer wieder Trockenjahre gab, in denen Wasser aus der Spree in 


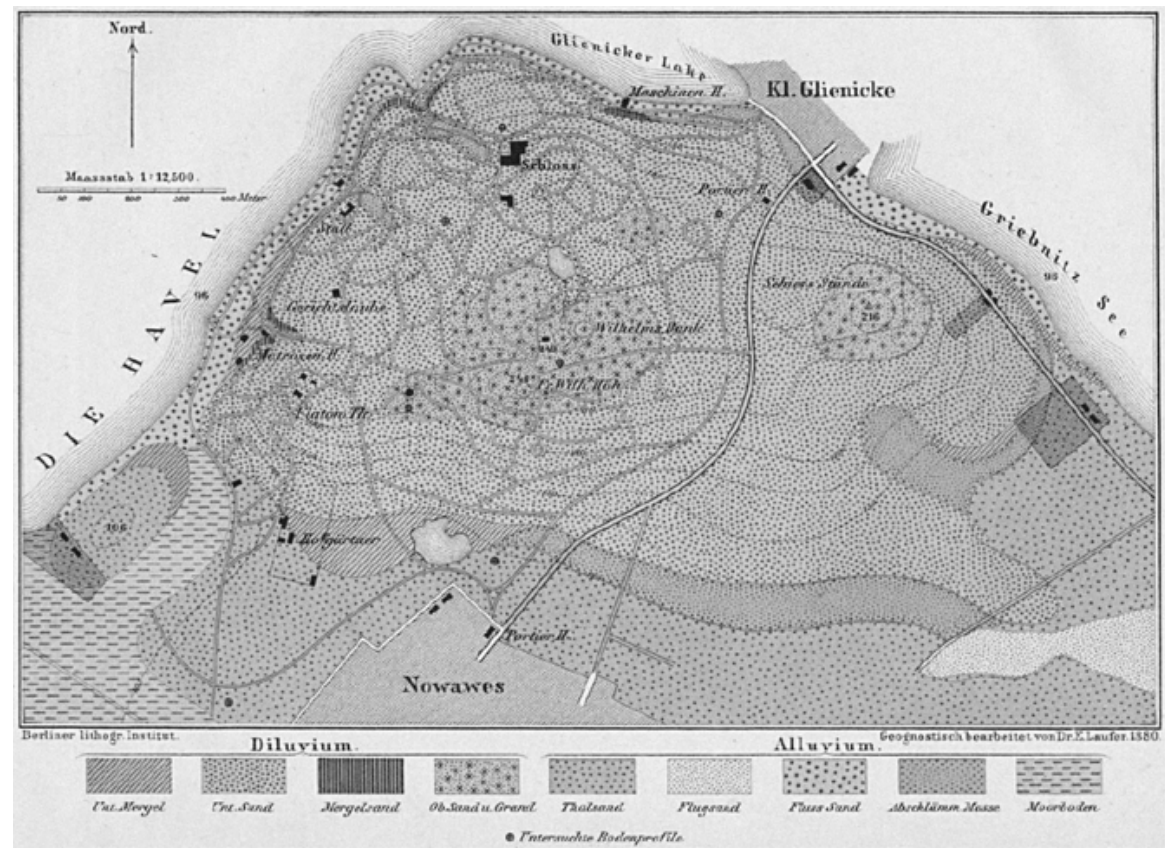

4 Geologisch-bodenkundliche Übersicht zum Park Babelsberg. In: Laufer 1881, 294.

den Park geleitet wurde, um das Wasserdefizit auszugleichen und Schäden am Baumbestand zu vermeiden.

Auf den teils durch Grundwasser, teils durch Stauwasser beeinflussten Flächen haben die Wurzeln der Gehölze durchweg Anschluss an das Grundwasser. Bei Grundwasserentzug sind die gehäuft auftretenden schwebenden wasserführenden Horizonte über bindigen Stauschichten für die Wasserversorgung der Bäume überlebensnotwendig. Auch die Dichtlagerung von Tonen und Schluffen kann sich durch den Überstau günstig auf die Wasserversorgung in Trockenphasen auswirken.

\section{Der Park Babelsberg}

Ein im Jahre 1880 erarbeiteter Beitrag unter dem Titel Der Babelsberg. Geognostisch und pedologisch bearbeitet von Herrn Ernst Laufer in Berlin (Laufer 1881) liefert einen ersten Einblick in die Typologie und Nährstoffversorgung von Böden im Park Babelsberg (Abb. 4).

Allerdings waren diese Angaben nur eingeschränkt flächenrepräsentativ. Im Jahre 2004 legten Eisbein/Wacker (2004) eine Studie unter dem Titel Drei Maßnahmen der Garten- 


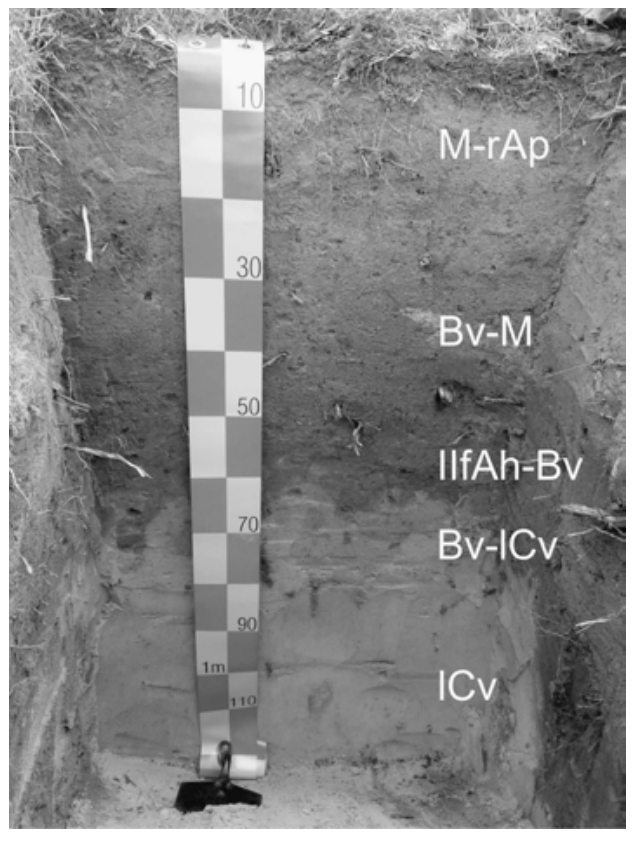

5 Bodenprofil mit ca. $120 \mathrm{~cm}$ Aufschlusstiefe am Hang nördlich unterhalb des Schlosses Babelsberg mit kolluvialen Bodenhorizonten (M-rAp, Bv-M), die einen begrabenen Braunerde-Rest inklusive der alten Geländeoberfläche (IIfAh-Bv, Bv-lCv) überlagern. Das Bodenprofil weist damit am selben (!) Standort sowohl Merkmale der Erosion (Kappung einer Braunerde, älter) als auch der Akkumulation (Überlagerung durch ein Kolluvium, jünger) auf und verweist auf eine differenzierte Nutzungsdynamik und Bodengenese bereits vor der Anlage des Parks Babelsberg.

denkmalpflege im Park Babelsberg vor. Darin berichten die Autoren über die vielfältigen Störungen der natürlichen Bodenstruktur durch Eingriffe in den Boden sowie durch Aufschüttung von Fremdmaterial. Aus dieser Untersuchung wird ersichtlich, dass ein beträchtlicher Teil der Flächen anthropogene bzw. stark anthropogen überprägte Böden aufweist. Dazu gehören Aushübe, Aufschüttungen, Auffüllungen sowie die Applikation von Komposten mit teils flächenhafter Ausdehnung und vielfach in mehrfacher Wiederholung. So wurde u.a. das durch Kriegs- und Nachkriegseingriffe gestörte Bodenrelief wieder ausgebessert. Im frühen 20. Jahrhundert wurde hinter dem Schloss sogar Boden ausgehoben und in der Nähe des Kindermannsees wieder aufgetragen. Generell sind auch in der Zukunft Bodenmodellierungen geplant. Hinzu kommen anthropogen verursachte Bodenveränderungen, die aus der Vornutzung des Gartenareals insbesondere seit dem Mittelalter herrühren. Als ein Gebiet mit teilweise ackerbaulicher Nutzung finden sich im Park Bodenprofile, die eine erhebliche Bodenerosion bzw. -akkumulation nahelegen (Abb. 5). Aufgrund dieser starken anthropogenen Überprägung kann nicht allein von topographischen und geologischen Informationen auf die Bodenverbreitung im Park Babelsberg geschlossen werden.

Aktuelle Informationen zu den Bodentypen im Park Babelsberg und ihren chemischen und physikalischen Eigenschaften finden sich im Online-Dienst der Bundesanstalt für Geowissenschaften und Rohstoffe/BGR (LGBR 2018). Hierbei handelt es sich um Ableitungen aus großmaßstäblichen Informationen, die kleinräumig allerdings nur näherungsweise Schätzungen zulassen. Danach überwiegen am Standort des Schlosses Babelsberg podso- 
lige Braunerden und Podsol-Braunerden aus Sand über Schmelzwassersand und, gering verbreitet, aus kiesführendem Sand über Schmelzwassersand. Vereinzelt sind Podsole und Braunerde-Podsole aus Sand über Schmelzwassersand sowie lessivierte Braunerden aus Sand über Lehmsand oder Lehm zu finden. Als weitere Bodentypen mit geringer Ausbreitung sind vergleyte Braunerden aus Sand über Urstromtal- oder Schmelzwassersand, auf Versiegelungsflächen Lockersyroseme und Pararendzinen aus grus- und schuttführendem Kippcarbonatsand mit Bauschutt über sehr tiefem Schmelzwasser- oder Urstromtalsand zu finden. Auch Hortisole, Regosole und Kolluvisole aus grusführendem Kippsand mit Bauschutt über tiefem Schmelzwasser- oder Urstromtalsand treten vereinzelt auf. Ebenfalls vereinzelt sind Gley-Braunerden und vergleyte Braunerden aus Sand über Urstromtalsand anzutreffen.

Zu den Böden des Parks Babelsberg liegen weder Nährstoff-/Kohlenstoffanalysen noch $\mathrm{pH}-$ Messungen vor. Aufgrund der bodentypologischen Hinweise (s.o.) ist zu vermuten, dass an diesem Standort sehr nährstoffarme Böden mit geringer Wasserkapazität vorherrschen. Aus diesem Grund wird aktuell mit Kompost gedüngt, um den Humusgehalt und damit die Nährstoffversorgung und Wasserkapazität zu verbessern.

Der Grundwasserstand liegt ungefähr bei $30 \mathrm{~m}$ NHN. Die vorliegenden Messungen stammen von nahegelegenen Messstellen außerhalb des Parks. Das heißt, an manchen Stellen im Park könnten die Wurzelsysteme der Bäume über einen Grundwasseranschluss verfügen. Da der Park Babelsberg aber sehr hügelig ist und sich bis ungefähr $78 \mathrm{~m} \mathrm{NHN} \mathrm{erhebt,}$ variieren die Grundwasser-Flurabstände sehr stark. Daher dürften die Gehölze in diesem Park überwiegend auf grundwasserfernen Arealen stocken. Zudem sind die Böden aufgrund ihres Ausgangsmaterials sehr wasserdurchlässig. An vereinzelten Stellen könnten Lehmanteile und alte Humushorizonte die Wasserspeicherkapazität erhöhen. Lokal sind die Böden drainiert.

Anders als im Dessau-Wörlitzer Gartenreich und im Branitzer Park fehlt in Babelsberg eine systematische bodenkundliche Kartierung des Parkgeländes. Die topographischen und geologischen Informationen reichen hier aufgrund der Inhomogenität der Böden nicht aus, um Rückschlüsse auf die Bodeneigenschaften zu ziehen und standortspezifische Bewirtschaftungsmaßnahmen abzuleiten.

\section{Schlussfolgerungen}

Die dargestellten historischen Gärten liegen im Übergangsbereich vom ozeanisch zum kontinental geprägten Klima in Deutschland mit ausgeprägten sommerlichen Trockenperioden. Diesen besonderen Klimaverhältnissen wurde bei der Anlage der Gärten bereits Rechnung getragen. Es ist jedoch fraglich, ob die damals getroffenen Maßnahmen angesichts der aktuellen Klimaentwicklungen auch weiterhin einen ausreichenden Schutz bieten.

Die relativ umfänglichen bodenkundlichen und hydrologischen Daten zum DessauWörlitzer Gartenreich und zum Park Branitz sind ein eindrücklicher Beleg dafür, dass die 
aus der jeweiligen Landschaftsgenese entstandene standortbezogene Heterogenität durch vielfältige anthropogene Einflüsse und vor allem durch gestalterische Eingriffe überprägt und dadurch der kleinräumige Wechsel von Substrateigenschaften zusätzlich verstärkt wurde. Die hier vorgestellten Ergebnisse der verschiedenen Bodenkartierungen zeigen, dass ein Vergleich dieser Standorte nur durch eine Systematisierung der Bodenansprache entsprechend den Vorgaben der aktuellen bodenkundlichen Kartieranleitung (Ad-hoc-AG Boden 2005) gelingen kann. Die Erfassung der räumlichen Heterogenität der Bodenverhältnisse spielt wiederum für Entscheidungen in der gärtnerischen Denkmalpflege eine erhebliche Rolle, so z.B. bei der Auswahl standörtlich angepasster Pflanzen und bei der gezielten Durchführung meliorativer Maßnahmen (Düngung, Mulchen, Einarbeitung organischer Substanz etc.).

Für die systematische Erfassung, Darstellung und Analyse von Böden und ihrer Heterogenität bietet sich die Nutzung eines Geographischen Informationssystems (GIS) an. Es ermöglicht die fortlaufende Ergänzung von Informationen zu Pflegemaßnahmen, lässt sich mit Informationen zu den chemischen und physikalischen Bodeneigenschaften sowie zum Ernährungszustand der Vegetation ergänzen und könnte mit Hilfe moderner Sensorik zu einem effizienten Monitoring-Instrument entwickelt werden, um schließlich den Ressourceneinsatz (Wasser, Nährstoffe, Arbeitszeit) zu steuern und Entscheidungen bei der Gartenplanung zu unterstützen und zu dokumentieren. Dies gilt insbesondere im Hinblick auf präventive Maßnahmen zur Klimaanpassung und zur raschen und räumlich expliziten Intervention bei kurzfristig wirksam werdenden Klimaextremen. Ein weiterer sinnvoller Schritt wäre die Ergänzung eines solchen Informationssystems mit historischen Daten, z.B. zur präzise datierten Entwicklung der Bodendecke und der dadurch ableitbaren Nutzungsgeschichte inklusive der Park-Vornutzung. Die Verfügbarkeit georeferenzierbarer Informationen zu Flora, Fauna, Boden und Wasser und ihre Verknüpfung mit historischen Informationen stellt ein Desiderat der meisten größeren Parks dar und kann zu einem wichtigen verbindenden Element zwischen den Parkverwaltungen werden.

\section{Literaturverzeichnis}

Ad-hoc-AG Boden $\left(2005^{5}\right)$ : Bodenkundliche Kartieranleitung. Stuttgart: Schweizerbart.

Appel, Lena (2016): Bodenverhältnisse in den Historischen Gärten Branitz, Babelsberg \& Großer Tiergarten. Dokumentation im Auftrag der interdisziplinären Arbeitsgruppe »Historische Gärten im Klimawandel« der Berlin-Brandenburgischen Akademie der Wissenschaften.

Cubasch, Ulrich; Kadow, Christopher (2011): Global climate change and aspects of regional climate change in the Berlin-Brandenburg region. In: Die Erde 142, 3-20.

Deutscher Klimaatlas (2018): https://www.dwd.de/DE/klimaumwelt/klimaatlas/klimaatlas_node.html?selected SektorId=3 (09.01.2019).

Eisbein, Karl; Wacker, Jörg (2004): Drei Maßnahmen der Gartendenkmalpflege im Park Babelsberg. In: Jahrbuch Stiftung Preußische Schlösser und Gärten Berlin-Brandenburg 6, 149-172.

Fintelmann, Gustav Adolf (1841): Die Wildbaumzucht, Die Zucht und Pflege der in Deutschland im freien Lande $z u$ erziehenden und zu überwinternden Holzpflanzen, sowohl der harten als auch der zarten. Berlin: Verlag Friedrich August Herbig. 
Hüttl, Reinhard; Bellmann, Klaus; Seiler, Wolfgang (Hg.) (1995): Atmosphärensanierung und Waldökosysteme: SANA: Wissenschaftliches Begleitprogramm zur Sanierung der Atmosphäre über den neuen Bundesländern. Wirkungen auf Kiefernbestände. Taunusstein: Verlag E. Blottner.

Jäger, Hermann (1865): Die Ziergehölze der Gärten und Parkanlagen. Weimar: Voigt.

Kulturstiftung DessauWörlitz (2005): Gehölze und ihre Standortbedingungen im Dessau-Wörlitzer Gartenreich. Modellvorhaben zur Erfassung von Wechselwirkungen zur Gefahrenabwehr für den Gehölzbestand in den Wörlitzer Anlagen und im Luisium. Dessau.

LBGR - Landesamt für Bergbau, Geologie und Rohstoffe Brandenburg (2018). Onlinedienst: http://www.geo. brandenburg.de/boden (09.01.2019).

Laufer, Ernst (1881): Der Babelsberg. Geognostisch und pedologisch bearbeitet von Herrn Ernst Laufer in Berlin. In: Jahrbuch der königlichen Preussischen geologischen Landesanstalt und Bergakademie 1, 294-334.

Meyer, Johann Heinrich Gustav $\left(1873^{2}\right)$ : Lehrbuch der schönen Gartenkunst mit besonderer Rücksicht auf die praktische Ausführung von Gärten und Parkanlagen. Berlin: Verlag von Ernst \& Korn.

Rippl, Helmut; Gockel, Gert; Neumann, Eckhard E. (2013): Das Verhalten von Bäumen unter bergbaulicher Grundwasserabsenkung: Ergebnisse 30-jähriger Beobachtungen. Cottbus: Förderverein Kulturlandschaft Niederlausitz.

\section{Bildnachweis}

1 Datenbasis: Deutscher Wetterdienst, eigene Elemente ergänzt; 2 Foto: Claudius Wecke, Stiftung Fürst-Pückler-Museum Park und Schloss Branitz; 3 Rippl et al. 2013, 178, Abb. 339; 4 Laufer 1881, 294, Abb. 1; 5 Profilaufnahme und Foto: Knut Kaiser, Juni 2018. 